\title{
Evaluación de Errores de Posicionamiento en los 6 Grados de Libertad en Pacientes con Cáncer de próstata tratados con radioterapia
}

\section{Evaluation of Positioning Errors in the 6 Degrees of Freedom in Patients with Prostate Cancer Treated with Radiotherapy}

\author{
Ricardo Antonio Sanchez Forero ${ }^{1}$ Peter Alfonso Olejua Villa ${ }^{1}$ Andrea Rocha Morales ${ }^{1}$ Raul Murillo ${ }^{1}$ \\ ${ }^{1}$ Hospital Universitario San Ignacio, Bogotá, Colombia \\ Urol Colomb 2021;30:23-33. \\ Address for correspondence Ricardo Antonio Sanchez Forero, \\ Oncologo Radioterapeuta, Hospital Universitario San Ignacio, Bogotá, \\ Colombia (e-mail: rsanchez3004@yahoo.com).
}

\begin{abstract}
Resumen
Palabras clave

- radioterapia

- cáncer de próstata

- error de posicionamiento

- márgenes
\end{abstract}

\section{Abstract}

Objetivos Determinar la magnitud de los errores de posicionamiento en los 6 grados de libertad y calcular el Margen a CTV para un protocolo adaptado en pacientes con cáncer de próstata temprano y localmente avanzado en un centro de referencia en Colombia.

Métodos Se realizó un estudio observacional prospectivo en 61 pacientes con cáncer de próstata tratados con radioterapia en el Centro Javeriano de Oncología del Hospital Universitario San Ignacio durante el 2018, con toma de imágenes volumétricas de rayos $x$ durante los primeros 3 días de tratamiento obteniéndose un promedio en errores traslacionales y rotacionales. Posteriormente se tomaron imágenes semanales. Además, se realizó el cálculo del margen al PTV y variaciones en vejiga y recto durante el tratamiento. Resultados Un total de 508 imágenes fueron registradas durante las 8 semanas de tratamiento, los errores traslacionales y rotacionales tuvieron un comportamiento alrededor de cero sin diferencias significativas inclusive en pacientes con IMC $>=25$. También se calculó el margen al PTV encontrándose entre 5 y $8 \mathrm{~mm}$, en cuanto a las medidas de vejiga y recto no se encontraron diferencias estadísticamente significativas en las imágenes adquiridas durante la radioterapia.

Conclusiones De acuerdo a los datos encontrados en este estudio, una adecuada reproducibilidad en paciente con cáncer de próstata puede ser encontrada usando el método de registro rígido de la anatomía pélvica ósea, promediando las 3 primeras imágenes y posteriormente con imágenes semanales, en centros con alta demanda de pacientes garantizando así una adecuada precisión del tratamiento.

Objectives To determine the magnitude of the positioning errors in the 6 degrees of freedom and calculate the Margin to CTV for an adapted protocol in patients with early and locally advanced prostate cancer in a reference center in Colombia. received January 21, 2020 accepted June 17, 2020 published online January 13, 2021
DOI https://doi.org/ $10.1055 / \mathrm{s}-0040-1714726$ ISSN 0120-789X. e ISSN 2027-0119.
(C) 2021. Sociedad Colombiana de Urología. All rights reserved. This is an open access article published by Thieme under the terms of the Creative Commons Attribution-NonDerivative-NonCommercial-License, permitting copying and reproduction so long as the original work is given appropriate credit. Contents may not be used for commercial purposes, or adapted, remixed, transformed or built upon. (https://creativecommons.org/ licenses/by-nc-nd/4.0/)

Thieme Revinter Publicações Ltda., Rua do Matoso 170, Rio de Janeiro, RJ, CEP 20270-135, Brazil 


\section{Keywords \\ - radiation therapy \\ - prostate cáncer \\ - positioning error \\ - margins}

Methods A prospective observational study was performed in 61 prostate cancer patients treated with radiotherapy at the Javeriano Oncology Center of the San Ignacio University Hospital during 2018, with volumetric X-ray imaging during the first 3 days of treatment obtaining a average in translational and rotational errors. Subsequently, weekly images were taken. In addition, the calculation of the margin to the PTV and variations in bladder and rectum during the treatment was performed.

Results A total of 508 images were recorded during the 8 weeks of treatment, translational and rotational errors had a behavior around zero without significant differences even in patients with BMI $>=25$. The margin to the PTV was also calculated between 5 and $8 \mathrm{~mm}$, in terms of bladder and rectum measurements, no statistically significant differences were found in the images acquired during radiotherapy.

Conclusions According to the data found in this study, an adequate reproducibility in a patient with prostate cancer can be found using the method of rigid registration of the pelvic bone anatomy, averaging the first 3 images and subsequently with weekly images, in centers with high patient demand guaranteeing adequate treatment accuracy.

\section{Introducción}

En Colombia se presentan cerca de 12.712 casos nuevos por año de cáncer de próstata y es igualmente el cáncer más frecuente entre los hombres. ${ }^{1}$

El tratamiento del cáncer de próstata localizado puede ir desde la vigilancia activa, prostatectomía radical, radioterapia y/o hormonoterapia. ${ }^{2,3}$ Avances tecnológicos en radioterapia en las últimas dos décadas, en especial con la implementación de técnicas de radioterapia de intensidad modulada (IMRT-por sus siglas en inglés) y arcoterapia volumétrica modulada (VMAT- por sus siglas en inglés), han permitido el escalonamiento de la dosis de radiación suministrada a la próstata, mostrando mejores resultados en supervivencia libre de falla bioquímica en paciente tratados con dosis altas. ${ }^{4,5}$

Dado que esas técnicas realizan distribuciones de dosis más precisas en el volumen blanco, es necesario un procedimiento con mayor precisión para el suministro del tratamiento. Las incertidumbres del posicionamiento y los movimientos del órgano (inter e intrafracción), son los dos factores contribuyentes más importantes para la precisión cuando se realiza tratamiento con radioterapia externa, ya que ellos pueden causar el suministro de una dosis subóptima en el tumor o aumentar la dosis en los órganos a riesgo. ${ }^{6}$ Por tanto, el volumen blanco clínico (CTV- por sus siglas en inglés de Clinical Target Volume), requiere de márgenes de seguridad que garanticen el cubrimiento adecuado del volumen planeado (PTV- por sus siglas en inglés de Planning Treatment Volume).

La radioterapia guiada por imágenes (IGRT-por sus siglas en inglés) ha mejorado la precisión dado que permite evaluar el volumen tridimensional además de dar información sobre los tejidos blandos, y de esa manera, permite además cuantificar y corregir los errores de posicionamiento, definido como la diferencia de la posición del paciente entre la tomografía de simulación y la posición en la máquina antes de suministrar el tratamiento, existen errores traslacionales (medidos en milímetros) que son los que se presentan en las coordenadas $\mathrm{X}, \mathrm{Y}, \mathrm{Z}$ del plano cartesiano y que corresponden a los desplazamientos en los eje axial, coronal y sagital en el paciente y los otros errores son rotacionales (medidos en grados) es decir, las desviaciones en rotación del paciente, bien sea en estructuras en la pelvis ósea o estructuras internas como la próstata y vesícula seminales. No obstante, esos últimos han sido poco estudiados para definir su verdadero impacto en el suministro de la dosis de radiación. ${ }^{7-9} \mathrm{El}$ estándar de oro es la obtención de imágenes diarias realizando correcciones del posicionamiento antes de suministrar el tratamiento ${ }^{9}$; sin embargo, dados los altos volúmenes de pacientes en máquina y la disponibilidad variable de tecnología, se han planteado alternativas como la obtención de imágenes bidimensionales (con o sin fiducias), o técnicas de imágenes volumétricas de rayos $\mathrm{x}$ (por sus siglas en inglés XVI - CBCT), fuera de línea que implican la obtención de imágenes en los primeros días de tratamiento $\mathrm{y}$ posteriormente de forma semanal.

Por otra parte, se ha descrito que pacientes muy delgados, así como pacientes con obesidad, presentan rotación de la pelvis ósea durante la tomografía de simulación induciendo a errores sistemáticos durante el tratamiento. ${ }^{10}$ El objetivo del presente estudio, es describir los errores de posicionamiento traslacionales y rotacionales y la reproducibilidad de los órganos a riesgo (vejiga y recto), así como evaluar los márgenes al CTV (PTV) en la rutina del tratamiento de pacientes con la modificación descrita del protocolo, en un centro de referencia en Colombia.

\section{Métodos}

Se realizó un estudio prospectivo observacional, en un grupo de 61 pacientes con cáncer de próstata temprano y localmente avanzado, del Centro Javeriano de Oncología- Hospital 
Universitario San Ignacio en Bogotá-Colombia, durante el año 2018, previa autorización del Comité de Investigaciones y Ética de la facultad de medicina de la Universidad Javeriana, manteniendo la total adherencia a los principios éticos y científicos.

Se calculó un tamaño de muestra de 61 sujetos para la determinación de un promedio de $1 \mathrm{~mm}$ en errores traslacionales con confianza de 95\%, desviación estándar y amplitud del intervalo de confianza de 0,5.

\section{CT Simulación y Planeación}

Los pacientes fueron colocados en posición supina con inmovilización en región poplítea e interpedia. Se realizaron cortes tomográficos de espesor de tres milímetros desde L3 hasta cuatro centímetros por debajo de las tuberosidades isquiáticas, con vejiga llena y recto vacío. Tres marcadores radio-opacos fueron colocados en piel (anterior y laterales derecho e izquierdo) para establecer el corte fiducial y fueron tatuados en esa posición. Marcas fiduciales dentro de la próstata no fueron usadas en ese grupo de pacientes.

Las imágenes fueron enviadas al sistema de la planeación Mónaco (versión 5.11.02), en donde se realizó el contorno de los volúmenes de tratamiento: próstata en bajo riesgo, próstata más base de vesículas en riesgo intermedio y próstata más la totalidad de las vesículas seminales en pacientes de alto riesgo (CTV) y órganos a riesgo (vejiga, recto y fémures), posteriormente se dio expansión al CTV de $10 \mathrm{~mm}$ en sentido cráneo caudal, transversal y anterior con disminución a $8 \mathrm{~mm}$ en sentido posterior (PTV). La planeación se realizó en todos los pacientes con técnica VMAT, con algoritmo de fotón - Montecarlo, grilla de cálculo de dos milímetros y energía de $6 \mathrm{MeV}$, mediante un arco de ida y vuelta de 320 grados en cada sentido, iniciando en 200 grados e incluyendo la camilla para efectos de atenuación y dispersión del haz de radiación.

El tratamiento de radioterapia se realizó en acelerador lineal ELEKTA - AXESSE con camilla Hexapod $®$, que permite 6 grados de libertad.

\section{Adquisición de Imágenes XVI y Corrección}

Se inició el procedimiento con una frecuencia diaria en las tres primeras sesiones, para permitir establecer un valor promedio de los errores de posicionamiento y posteriormente se continuó con una imagen semanal por cada paciente durante las siguientes 7 semanas del curso de tratamiento.

La adquisición de imágenes se realizó emulando la posición de simulación. Mediante protocolo de imágenes volumétricas (XVI o llamada CBCT), próstata M15, con los siguientes factores técnicos: Arco entre -180 grados y 180 grados CW y 660 fotogramas para reconstrucción, la cual es comparada con la serie tomográfica de simulación que contiene los contornos del volumen blanco y órganos a riesgo y las coordenadas de isocentro definidas en el sistema de planeación. Posteriormente se realizó el registro del área de interés con superposición del conjunto de imágenes para ser alineadas de forma automática y ajustar los valores de grises y/o estructuras óseas, permitiendo comparar la posición de las estructuras óseas de la pelvis tanto traslacionales como rotacionales en las coordenadas $\mathrm{X}$, Y, Z y poder realizar la corrección antes del tratamiento.

\section{Cálculo del Margen CTV-PTV}

Para el cálculo del margen MPTV, se toman los errores traslacionales de la semana 2 a la 8 . Por ende, el margen es calculado para correcciones semanales y no diarias. El supuesto es que los errores no varían de una sesión a otra. Para corroborar ese supuesto se utilizó la prueba de Friedman. Se calculó dicho margen mediante la fórmula: $\mathrm{MPTV}=2.5 \Sigma+0.7 \sigma$, propuesta por Van Herk y col. (10,Gleeson, 2014).

\section{En Donde}

$\Sigma$ : La desviación estándar de los errores sistemáticos, se estima como la desviación estándar de los promedios de los errores traslacionales por paciente.

$\sigma:$ La desviación estándar de los errores aleatorios. Para su estimación, primero se calculan la desviación estándar de los errores traslacionales por paciente. Con esas desviaciones, se calcula entonces la raíz de la media cuadrática (RMS) (-Figura 1).

La fórmula se basa en la distribución de la dosis acumulada y mínima acumulada de CTV. Ella considera la influencia de ambos errores, sistemático y aleatorio, en donde la dosis mínima acumulada en el CTV puede alcanzar al menos el 95\% de la dosis prescrita en el $90 \%$ de los pacientes.

La fórmula de Van Herk fue aplicada para calcular los márgenes al PTV en las tres dimensiones. Se utilizaron los errores traslacionales crudos e imputando la media y la mediana por paciente. Los márgenes calculados utilizando los datos sin imputar fueron mayores a los calculados utilizando imputación. Con el objetivo de mantener la condición de alcanzar al menos el $95 \%$ de la dosis prescrita en el $90 \%$ de los pacientes, se seleccionan los márgenes calculados sin imputar por considerarse más seguros al cubrir mayor rango.

Para ver si hay diferencias en el MPTV por alguna de las covariables de interés, se realizaron regresiones lineales univariadas con el promedio y las desviaciones por pacientes como desenlaces. Para ver si había diferencia en el cálculo del MPTV por las medidas de esos órganos también se tomaron los promedios de esas medidas por paciente. La regresión multivariada en este caso no es de interés. Luego se realizó una prueba $\mathrm{F}$ para evaluar la significancia de las covariables. Al no encontrarse diferencias significativas por ninguna covariable se calculó el MPTV general y no por subgrupos.

Los errores rotacionales no se utilizan para el cálculo del MPTV, pero se describen y se analizó si existían diferencias por las covariables de interés. Al igual que en los errores traslacionales se realizó la prueba de Friedman para analizar diferencias entre una sesión y otra. Tanto para los errores traslacionales como para los rotacionales se tomaron los promedios por paciente y se realizó una prueba de rangos de signos de Wilcoxon para analizar si existía evidencia de descalibración de los errores. En todas las pruebas se consideró un error alfa de 0,05. 


\section{Errores traslacionales. Semana 1-8}
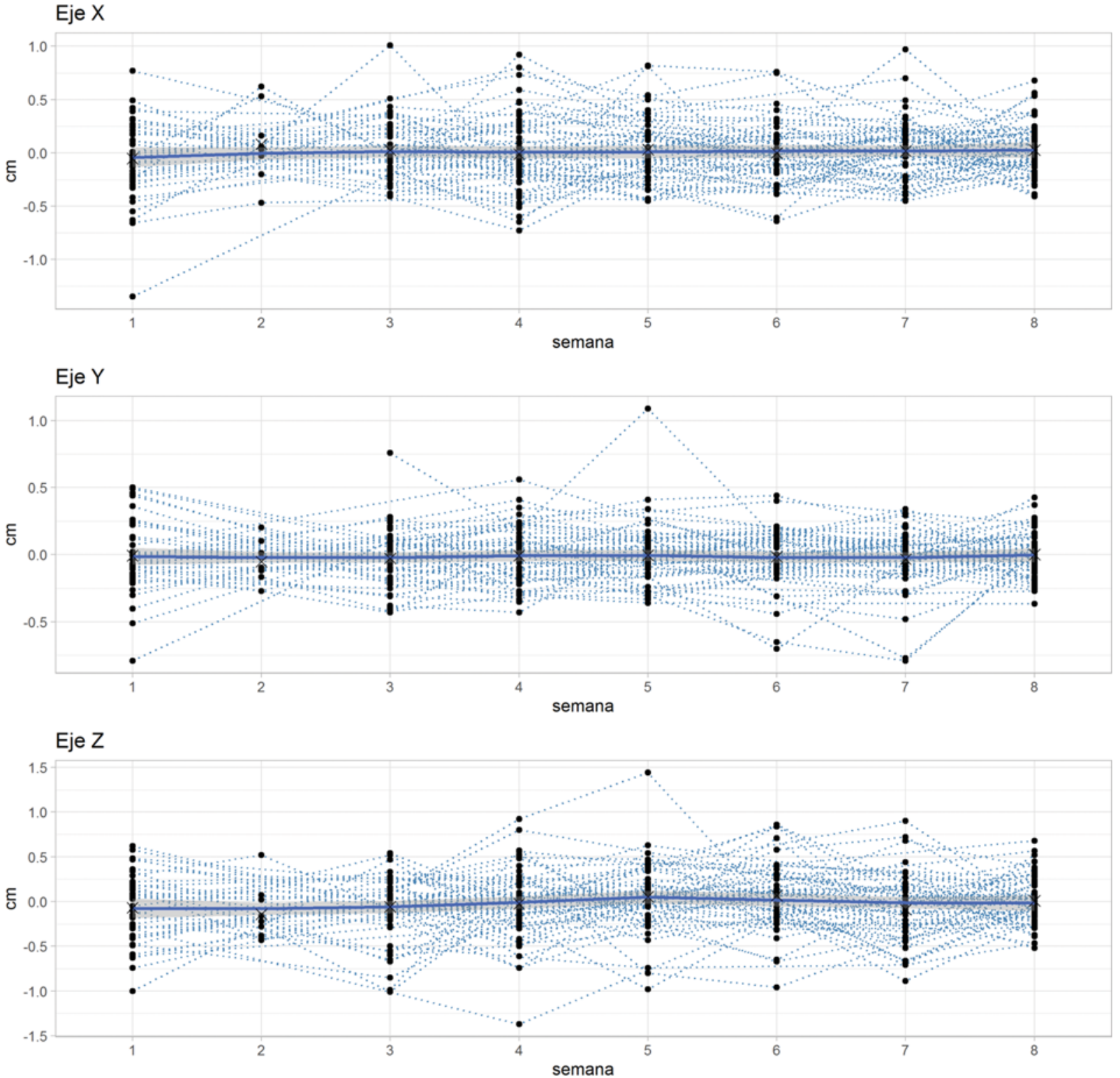

Los errores traslacionales en los ejes X, Y y Z la mediana se distribuyó alrededor de cero cm y la desviación estándar alrededor de $3 \mathrm{~mm}$.

Fig. 1 Errores traslacionales durante el tratamiento en los diferentes Ejes (X, Y y Z). Los errores traslacionales en los ejes $X, Y$ y $Z$ la mediana se distribuyó alrededor de cero $\mathrm{cm}$ y la desviación estándar alrededor de $3 \mathrm{~mm}$.

\section{Resultados}

Un total de 508 imágenes volumétricas de rayos $\mathrm{x}$, fueron registradas antes de suministrar el tratamiento durante 8 semanas en 61 pacientes. La mayoría de los pacientes tenían riesgo intermedio y alto. Las características demográficas y clínicas se describen en la - Tabla 1.

En la - Figura 1 se presentan los errores traslacionales en las tres dimensiones durante las 8 semanas de tratamiento. Tomando el mínimo y máximo por semana, el rango fue de $[-1,35,1,01]$ para el eje $X$; de $[-0,79,1,09]$ para el eje $Y$; y de $[-1,37,1,01]$ para el eje $Z$ (Anexo 1).

No se encontraron diferencias significativas entre las medidas axial, coronal y sagital en la vejiga. En la medida axial para el recto tampoco se encontraron diferencias significativas (Anexo 2).

En la - Tabla 2 se muestran los p valores correspondientes a la prueba $\mathrm{F}$ de cada covariable en los ejes X, Y y Z.

\section{Cálculo del MPTV}

En la - Tabla 3 se presentan los márgenes al PTV en los diferentes ejes utilizando los datos crudos.

\section{Errores Rotacionales}

En la - Figura 2 se muestran los errores rotacionales durante las 8 semanas de tratamiento. Se encontró sesgo en sentido positivo del eje $Y$, y en sentido negativo del eje $Z$, ambos estadísticamente significativos (Anexo 3). 
Tabla 1 Características de los pacientes

\begin{tabular}{|c|c|}
\hline & Total \\
\hline & $(n=61)$ \\
\hline \multicolumn{2}{|l|}{ Edad } \\
\hline Media (DE) & $68.8(5.56)$ \\
\hline Mediana [Min, Max] & $70.0[51.0,78.0]$ \\
\hline \multicolumn{2}{|l|}{ Peso } \\
\hline Media (DE) & $74.3(12.2)$ \\
\hline Mediana [Min, Max] & $71.0[55.0,108]$ \\
\hline \multicolumn{2}{|l|}{ Estatura } \\
\hline Media (DE) & $1.68(0.0671)$ \\
\hline Mediana [Min, Max] & $1.68[1.52,1.85]$ \\
\hline \multicolumn{2}{|l|}{ IMC } \\
\hline$<25$ & $29(47.5 \%)$ \\
\hline$>=25$ & $32(52.5 \%)$ \\
\hline \multicolumn{2}{|l|}{ Antígeno prostático específico (PSA) } \\
\hline Media (DE) & $13.2(9.58)$ \\
\hline Mediana [Min, Max] & $10.2[4.00,63.6]$ \\
\hline \multicolumn{2}{|l|}{ Gleason } \\
\hline 6 & $25(41.0 \%)$ \\
\hline 7 & $24(39.3 \%)$ \\
\hline$>=8$ & $10(16.4 \%)$ \\
\hline Sin clasificación & $2(3.3 \%)$ \\
\hline \multicolumn{2}{|l|}{ Tumor T } \\
\hline $\mathrm{T} 1$ & $18(29.5 \%)$ \\
\hline $\mathrm{T} 2$ & $17(27.9 \%)$ \\
\hline T3 & $5(8.2 \%)$ \\
\hline $\mathrm{T4}$ & $2(3.3 \%)$ \\
\hline Sin clasificación T (Tumor) & $19(31.1 \%)$ \\
\hline \multicolumn{2}{|l|}{ Riesgo } \\
\hline Bajo Riesgo & $12(19.7 \%)$ \\
\hline Riesgo Intermedio & $21(34.4 \%)$ \\
\hline Alto Riesgo & $23(37.7 \%)$ \\
\hline Muy Alto Riesgo & $1(1.6 \%)$ \\
\hline Sin clasificación de grupo de riesgo & $4(6.6 \%)$ \\
\hline IMC: Índice de Masa Corporal. & \\
\hline
\end{tabular}

La mayoría de los pacientes pertenecían al grupo de riesgo intermedio y de alto riesgo.

En la - Figura 3, muestra los errores de posicionamiento de acuerdo al IMC.

\section{Discusión}

Una de las fortalezas del estudio fue una adecuada reproducibilidad del tratamiento en pacientes con cáncer de próstata, siendo los errores de posicionamiento en los 6 grados de libertad (traslacionales y rotacionales) similares durante las 8 semanas. En relación a la variación de las
Tabla 2 P valores y variables

\begin{tabular}{|c|c|c|c|c|c|c|}
\hline \multirow[t]{2}{*}{ Variable } & \multicolumn{3}{|c|}{ Promedios } & \multicolumn{3}{|c|}{ Desviaciones } \\
\hline & Eje $X$ & Eje $Y$ & Eje Z & Eje $X$ & Eje $Y$ & Eje $Z$ \\
\hline \multicolumn{7}{|l|}{ Covariable } \\
\hline Peso & 0.97 & 0.4 & 0.38 & 0.28 & 0.69 & 0.37 \\
\hline Estatura & 0.78 & 0.44 & 0.93 & 0.46 & 0.6 & 0.38 \\
\hline IMC & 0.92 & 0.55 & 0.28 & 0.41 & 0.85 & 0.58 \\
\hline $\begin{array}{l}\text { IMC } \\
\text { categorizado }\end{array}$ & 0.84 & 0.16 & 0.16 & 0.42 & 0.34 & 0.17 \\
\hline \multicolumn{7}{|c|}{ Medida del órgano } \\
\hline Recto axial & 0.85 & 0.18 & 0.5 & 0.29 & 0.33 & 0.2 \\
\hline Vejiga axial & 0.91 & 0.05 & 0.39 & 0.28 & 0.74 & 0.69 \\
\hline $\begin{array}{l}\text { Vejiga } \\
\text { coronal }\end{array}$ & 0.96 & 0.33 & 0.1 & 0.91 & 0.31 & 0.66 \\
\hline $\begin{array}{l}\text { Vejiga } \\
\text { sagital }\end{array}$ & 0.35 & 0.18 & 0.63 & 0.3 & 0.46 & 0.63 \\
\hline
\end{tabular}

Abreviatura: IMC, Índice de Masa Corporal.

Tabla 3 Márgenes MPTV

\begin{tabular}{|l|l|l|l|l|}
\hline Eje & $\boldsymbol{\mu}$ & $\boldsymbol{\Sigma}$ & $\boldsymbol{\sigma}$ & Mptv \\
\hline $\mathrm{X}$ & 0 & 0.19 & 0.27 & 0.68 \\
\hline $\mathrm{Y}$ & -0.01 & 0.15 & 0.18 & 0.5 \\
\hline $\mathrm{Z}$ & -0.02 & 0.23 & 0.33 & 0.81 \\
\hline
\end{tabular}

El margen al PTV fue diferente en los tres ejes, siendo mayor en el Eje Z.

medidas axial, coronal y sagital en vejiga, y axial en recto, no se encontraron diferencias significativas; sin embargo, una limitación importante fue la medición lineal y no volumétrica de esos órganos.

La IGRT puede disminuir esas diferencias entre la posición de volumen blanco y órganos a riesgo visibles sobre las imágenes de planeación y durante el tratamiento. Existen 3 tipos de estrategias de corrección, la primera consiste en correcciones fuera de línea, en donde los ajustes se basan en imágenes adquiridas en fracciones previas, eso se traduce en un error sistemático medio, sin correcciones por variaciones diarias (error aleatorio). La segunda estrategia de corrección es en línea, en donde los ajustes son hechos inmediatamente después de la imagen; ella no solo corrige el error medio del paciente sino también las variaciones diarias. La tercera estrategia son las correcciones intrafracción, en donde múltiples imágenes y ajustes son hechos durante la fracción de radioterapia.

La primera estrategia de IGRT, que es la utilizada en el estudio, se basa en modelos biológicos y consideraciones físicas; específicamente toma en cuenta que el margen al CTV (PTV) es influenciado en su mayor parte por el error sistemático. ${ }^{11}$ Ese protocolo permite una reducción del margen con un limitado flujo de trabajo equivalente a un número de imágenes mínimo del 10\% de todas las fracciones. Debido a la carga de trabajo y la disponibilidad de recursos en el estudio, tomamos los 3 primeros días como base para el cálculo del promedio, lo que corresponde al 7,7\%, estando por debajo de lo recomendado; no obstante, los resultados 


\section{Errores rotacionales. Semana 1-8}
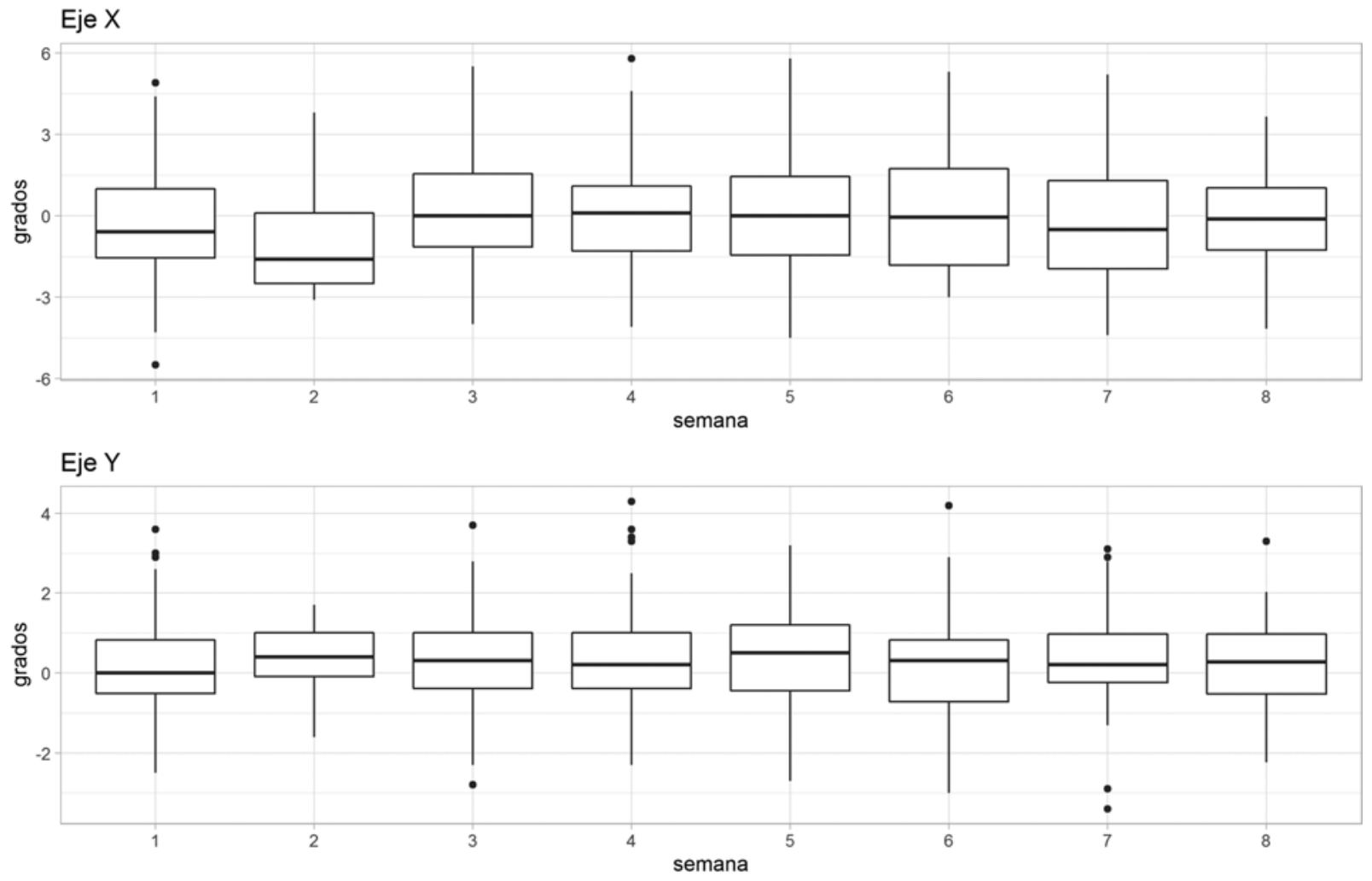

\section{Eje Z}

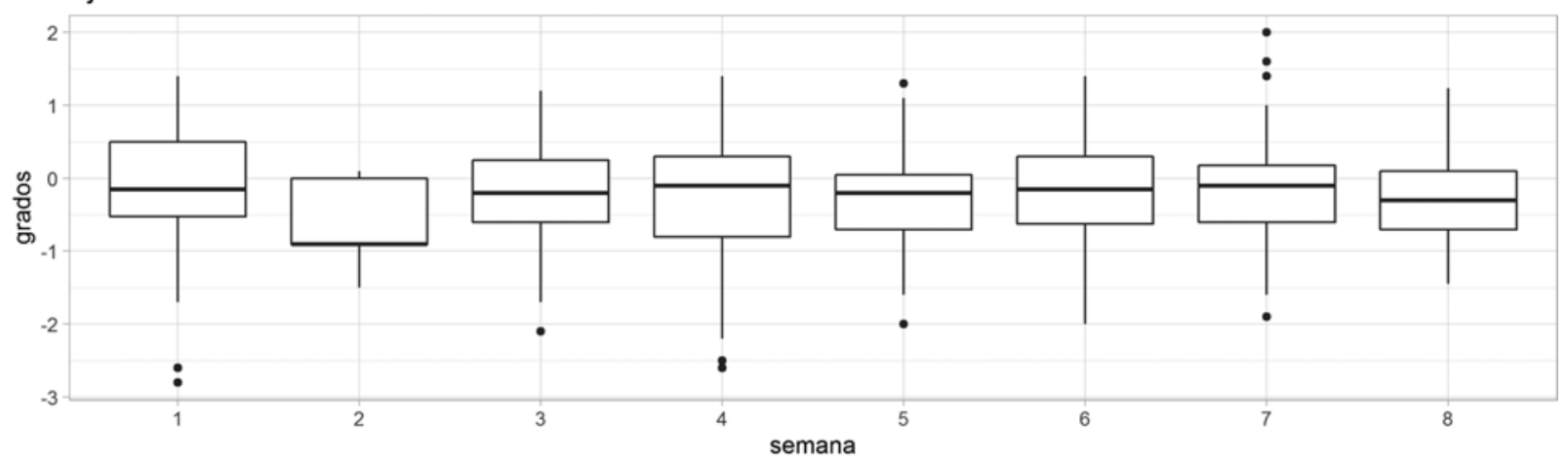

No se encontró diferencia significativa en los errores rotacionales durante las imágenes tomadas en las 8 semanas del tratamiento.

Fig. 2 Errores Rotacionales. No se encontró diferencia significativa en los errores rotacionales durante las imágenes tomadas en las 8 semanas del tratamiento.

obtenidos indican que no hay mayor variación frente a lo esperado, proporcionando márgenes adecuados para la seguridad del tratamiento. ${ }^{12}$

Basados en un número importante de imágenes volumétricas de tomografía computarizada de haz cónico (CBCT -XVI) realizadas a 61 pacientes, pudimos definir un margen PTV y además evaluar la variación del volumen en el recto y la vejiga durante todo el curso de la radioterapia.

Dentro de la variabilidad interfracción del recto, el riesgo más importante es la distensión, lo cual puede causar un impacto significativo desde el punto de vista dosimétrico y clínico en los pacientes con cáncer de próstata, en donde su zona periférica posterior, quedaría subdosificada, ${ }^{13,14}$ y estudios previos han mostrado mayor incidencia de falla bioquímica en los pacientes tratados con el recto distendido. ${ }^{15}$ De forma similar el volumen de la vejiga puede variar significativamente ${ }^{16}$; estudios previos con pacientes simulados con vejiga llena, muestran que durante el curso del tratamiento, ese volumen disminuye en el $90 \%$ de los casos, diferencia sistemática que podría impactar significativamente en la dosis sobre el volumen y aumentar su toxicidad.

En el presente estudio, encontramos escasa diferencia en el diámetro del recto comparado con la Tac de planeación, lo cual hace que se presente poca variación en la posición de la próstata. Se presentó mayor variación en las diferentes medidas de diámetros de la vejiga durante el curso del tratamiento, sin embargo, no fueron significativas con respecto a la Tac de planeación. 


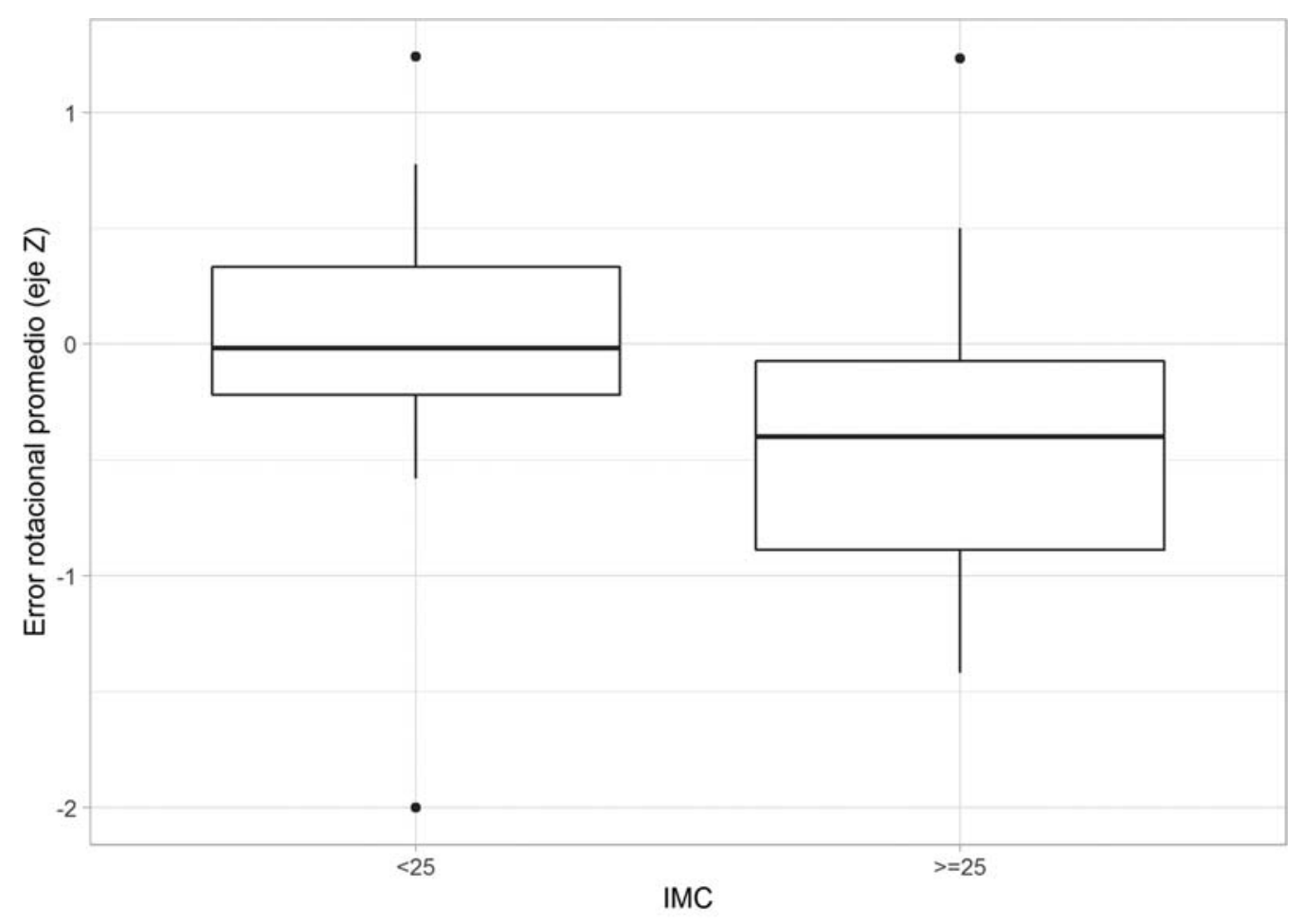

En el eje Z, fue mayor en pacientes con IMC >= 25, sin embargo, la diferencia no fue

estadisticamente significativa.

Fig. 3 Errores eje Z de acuerdo al IMC. En el eje Z, fue mayor en pacientes con IMC >=25, sin embargo, la diferencia no fue estadísticamente significativa.

La razón por la cual se presentó un menor desplazamiento en el eje Y, en especial en el eje Z, en comparación con el ejeX, es posiblemente la influencia de mantener durante el tratamiento las condiciones similares a la Tac de simulación. Estudios previos relacionados también han considerado que la vejiga llena y el recto vacío fueron los factores principales para disminuir las incertidumbres de posicionamiento. ${ }^{17,18}$

Además del desplazamiento lineal, la rotación ha sido estudiada durante el curso de tratamiento. Tehrani y col., ${ }^{19}$ reportaron con base en un algoritmo de punto más cercano (ICP), errores de rotación en los ejes centrales $X, Y, Z$ de $2,3^{\circ}$, $0,89^{\circ}$ y $0,72^{\circ}$, respectivamente. La razón del mayor error en el eje $\mathrm{X}$ es que él es más propenso para errores en traslación, y las diferencias significativas en esos dos tipos de errores pueden estar igualmente relacionadas con el estado de llenado de la vejiga y recto.

La rotación es de particular interés si las vesículas seminales están dentro del PTV. Como la próstata tiene una forma casi esférica, cuando se trata solo cáncer de próstata de bajo riesgo, el error de rotación no tiene importancia dado que el PTV no cambia significativamente en la dosis. Sin embargo, la adición de las vesículas seminales crea una PTV mucho más irregular, lo cual aumenta la importancia clínica de los errores de rotación. Como el 80,3\% de esta cohorte de pacientes incluyó las vesículas seminales para una parte o la totalidad de su curso de tratamiento, esta es una consideración importante; sin embargo, una de las limitaciones en el estudio fue la calidad de alineación realizada mediante registros rígidos de la pelvis ósea y no con la alineación de tejidos blandos ni fiducias implantadas, las que pueden brindar una mayor calidad en las correcciones rotacionales cuando las vesículas están incluidas en el PTV.

En pacientes obesos con cáncer de próstata tratados con radioterapia se ha encontrado mayor incertidumbre en el posicionamiento. Eso se debe, en parte, a la variabilidad en la ubicación de los marcadores externos de la piel de la pelvis (utilizados para configurar al paciente diariamente), en relación con la anatomía interna. Los grupos de pacientes con sobrepeso $\mathrm{y}$ obesos han mostrado diferencias significativas en la posición interfracción de la próstata. ${ }^{20}$ Nuestros hallazgos mostraron una tendencia de mayor error en el eje $z$ en pacientes con IMC $\geq 25$, que sin embargo no fue estadísticamente significativa ni en traslación ni en rotación. Es importante tener en cuenta que el tamaño de muestra limita la significancia estadística de los resultados.

De acuerdo a lo encontrado en este estudio, los márgenes al CTV podrían reducirse en un 20\%. Para mejorar en la precisión del volumen blanco, se podría implementar la alineación con tejidos blandos con o sin fiducias, sin embargo, ese procedimiento requiere mayor disponibilidad de personal, tiempo de máquina y en el caso de fiducias pasar a un procedimiento invasivo. No obstante, los resultados obtenidos con el protocolo analizado, indican que su implementación es posible con una adecuada reproducibilidad durante todo el curso de tratamiento y sin generar riesgo en el paciente, lo que resulta costo-efectivo ante condiciones de limitados recursos, situación frecuente en los centros de radioterapia de países en desarrollo. 


\section{Conclusiones}

Protocolos institucionales son requeridos para definir márgenes al CTV (PTV) basados en diferentes condiciones del suministro de la radioterapia. Es importante resaltar los pocos cambios en el volumen de la vejiga y el recto durante las imágenes del curso del tratamiento, lo cual impacta en la reproducibilidad del tratamiento de radioterapia.

\section{Conflicto de Intereses}

Los autores declaran no tener ningún conflicto de intereses.

\section{Referencias}

1 Bray F, Ferlay J, Soerjomataram I, Siegel RL, Torre LA, Jemal A. Global cancer statistics 2018: GLOBOCAN estimates of incidence and mortality worldwide for 36 cancers in 185 countries. CA Cancer J Clin 2018;68(06):394-424

2 Holmberg L, Bill-Axelson A, Helgesen F, et al; Scandinavian Prostatic Cancer Group Study Number 4. A randomized trial comparing radical prostatectomy with watchful waiting in early prostate cancer. N Engl J Med 2002;347(11):781-789

3 Bill-Axelson A, Holmberg L, Garmo H, et al. Radical prostatectomy or watchful waiting in early prostate cancer. N Engl J Med 2014; 370(10):932-942

4 Zelefsky MJ, Leibel SA, Gaudin PB, et al. Dose escalation with three-dimensional conformal radiation therapy affects the outcome in prostate cancer. Int J Radiat Oncol Biol Phys 1998; 41(03):491-500

5 Pollack A, Zagars GK, Starkschall G, et al. Prostate cancer radiation dose response: results of the M. D. Anderson phase III randomized trial. Int J Radiat Oncol Biol Phys 2002;53(05):1097-1105

6 Pouliot J, Bani-Hashemi A, Chen J, et al. Low-dose megavoltage cone-beam CT for radiation therapy. Int J Radiat Oncol Biol Phys 2005;61(02):552-560

7 Vences L, Wulf J, Vordermark D, Sauer O, Berlinger K, Roth M. Target motion measurement without implanted markers and its validation by comparison with manually obtained data. Med Phys 2005;32(11):3431-3439

8 Ma CM, Paskalev K. In-room CT techniques for image-guided radiation therapy. Med Dosim 2006;31(01):30-39

9 Létourneau D, Wong JW, Oldham M, et al. Cone-beam-CT guided radiation therapy: technical implementation. Radiother Oncol 2005;75(03):279-286
10 Sánchez R, González A, Rocha A. Reproducibility of patient positioning in radiation therapy Centro Javeriano de Oncología. Universitas Medica 2011;52(01):44-61

11 Bel A, Vos PH, Rodrigus PT, et al. High-precision prostate cancer irradiation by clinical application of an offline patient setup verification procedure, using portal imaging. Int J Radiat Oncol Biol Phys 1996;35(02):321-332

12 Amer AM, Mackay RI, Roberts SA, Hendry JH, Williams PC. The required number of treatment imaging days for an effective offline correction of systematic errors in conformal radiotherapy of prostate cancer-a radiobiological analysis. Radiother Oncol 2001; 61(02):143-150

13 Ost P, De Gersem W, De Potter B, Fonteyne V, De Neve W, De Meerleer G. A comparison of the acute toxicity profile between two-dimensional and three-dimensional image-guided radiotherapy for postoperative prostate cancer. Clin Oncol ( $R$ Coll Radiol) 2011;23(05):344-349

14 Ung NM, Wee L, Hackett SL, Jones A, Lim TS, Harper CS. Comparison of low-dose, half-rotation, cone-beam CT with electronic portal imaging device for registration of fiducial markers during prostate radiotherapy. J Appl Clin Med Phys 2013;14(04):4249

15 de Crevoisier R, Tucker SL, Dong L, et al. Increased risk of biochemical and local failure in patients with distended rectum on the planning CT for prostate cancer radiotherapy. Int J Radiat Oncol Biol Phys 2005;62(04):965-973

16 Frank SJ, Dong L, Kudchadker RJ, et al. Quantification of prostate and seminal vesicle interfraction variation during IMRT. Int J Radiat Oncol Biol Phys 2008;71(03):813-820

17 van Herk M, Remeijer P, Rasch C, Lebesque JV. The probability of correct target dosage: dose-population histograms for deriving treatment margins in radiotherapy. Int J Radiat Oncol Biol Phys 2000;47(04):1121-1135

18 Ten Haken RK, Forman JD, Heimburger DK, et al. Treatment planning issues related to prostate movement in response to differential filling of the rectum and bladder. Int J Radiat Oncol Biol Phys 1991;20(06):1317-1324

19 Tehrani JN, O'Brien RT, Poulsen PR, Keall P. Real-time estimation of prostate tumor rotation and translation with a $\mathrm{kV}$ imaging system based on an iterative closest point algorithm. Phys Med Biol 2013; 58(23):8517-8533

20 Wong JR, Gao Z, Uematsu M, et al. Interfractional prostate shifts: review of 1870 computed tomography (CT) scans obtained during image-guided radiotherapy using CT-on-rails for the treatment of prostate cancer. Int J Radiat Oncol Biol Phys 2008;72(05): 1396-1401 


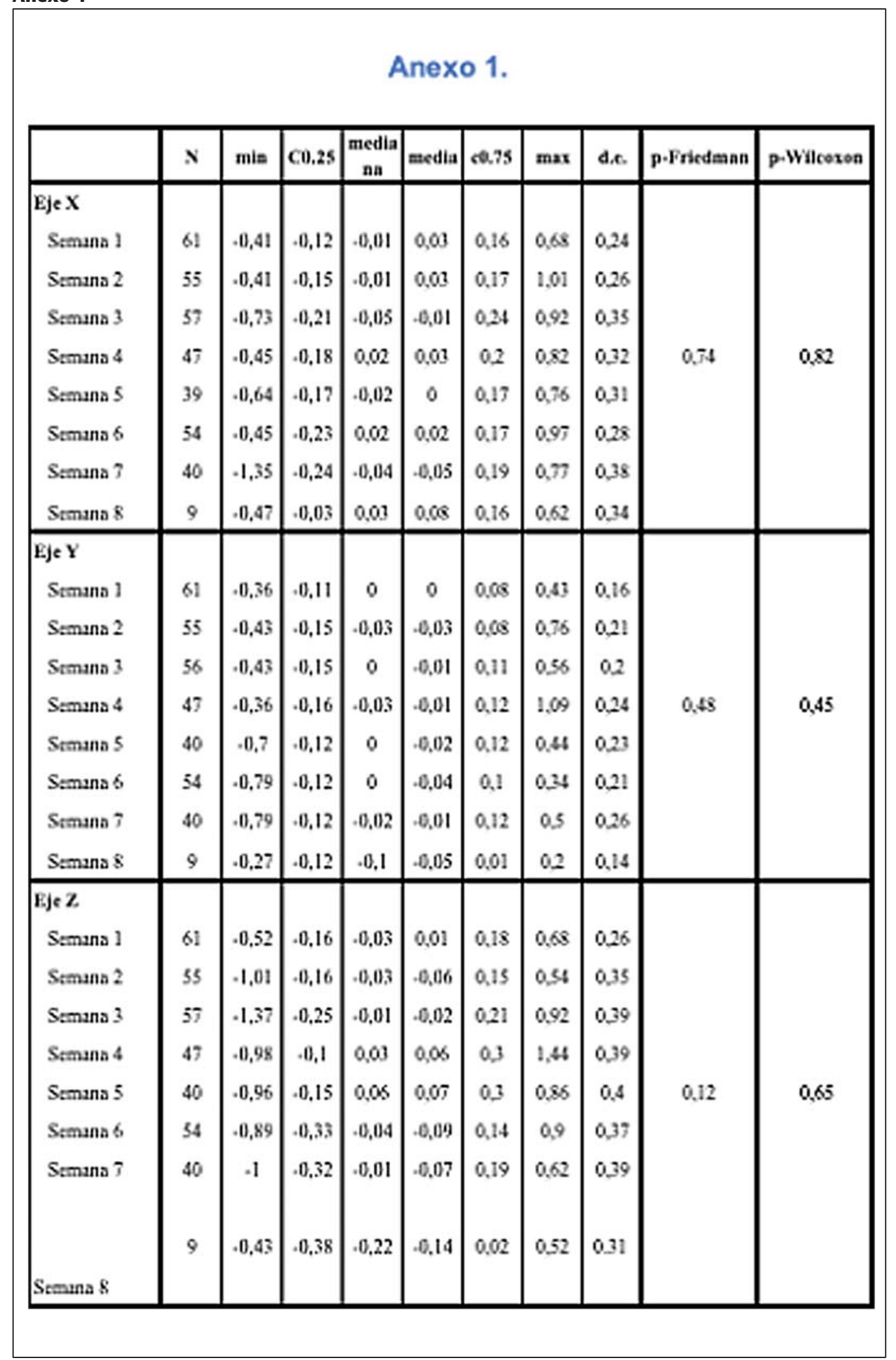


32 Evaluación de errores de posicionamiento en los 6 grados de libertad Sanchez Forero y col.

Anexo 2

\begin{tabular}{|c|c|c|c|c|c|c|c|c|c|c|}
\hline & $\mathrm{N}$ & $\min$ & $\mathrm{C} 0.25$ & mediana & media & c0.75 & $\max$ & d.e. & p-Friedman & p-Wilcoxon \\
\hline \multicolumn{9}{|l|}{ Eje $X$} & \multirow[t]{9}{*}{0,21} & \multirow[t]{9}{*}{0,69} \\
\hline Semana 1 & 61 & $-4,17$ & $-1,27$ & $-0,12$ & - & 1,03 & 3,65 & 1,84 & & \\
\hline Semana 2 & 55 & $-4,00$ & $-1,15$ & - & 0,15 & 1,55 & 5,50 & 2,14 & & \\
\hline Semana 3 & 57 & $-4,10$ & $-1,30$ & 0,10 & 0,10 & 1,10 & 5,80 & 2,04 & & \\
\hline Semana 4 & 47 & $-4,50$ & $-1,45$ & - & 0,12 & 1,45 & 5,80 & 2,57 & & \\
\hline Semana 5 & 40 & $-3,00$ & $-1,83$ & $-0,05$ & 0,21 & 1,72 & 5,30 & 2,24 & & \\
\hline Semana 6 & 54 & $-4,40$ & $-1,95$ & $-0,50$ & $-0,14$ & 1,30 & 5,20 & 2,36 & & \\
\hline Semana 7 & 40 & $-5,50$ & $-1,55$ & $-0,60$ & $-0,27$ & 1,00 & 4,90 & 2,28 & & \\
\hline Semana 8 & 9 & $-3,10$ & $-2,50$ & $-1,60$ & $-0,72$ & 0,10 & 3,80 & 2,36 & & \\
\hline \multicolumn{9}{|l|}{ Eje $Y$} & \multirow[t]{9}{*}{0,51} & \multirow[t]{9}{*}{0,03} \\
\hline Semana 1 & 61 & $-2,23$ & $-0,53$ & 0,27 & 0,24 & 0,97 & 3,30 & 1,09 & & \\
\hline Semana 2 & 55 & $-2,80$ & $-0,40$ & 0,30 & 0,22 & 1,00 & 3,70 & 1,31 & & \\
\hline Semana 3 & 57 & $-2,30$ & $-0,40$ & 0,20 & 0,42 & 1,00 & 4,30 & 1,33 & & \\
\hline Semana 4 & 47 & $-2,70$ & $-0,45$ & 0,50 & 0,34 & 1,20 & 3,20 & 1,19 & & \\
\hline Semana 5 & 40 & $-3,00$ & $-0,72$ & 0,30 & 0,19 & 0,82 & 4,20 & 1,56 & & \\
\hline Semana 6 & 54 & $-3,40$ & $-0,25$ & 0,20 & 0,34 & 0,98 & 3,10 & 1,23 & & \\
\hline Semana 7 & 40 & $-2,50$ & $-0,52$ & - & 0,20 & 0,82 & 3,60 & 1,35 & & \\
\hline Semana 8 & 9 & $-1,60$ & $-0,10$ & 0,40 & 0,38 & 1,00 & 1,70 & 1,03 & & \\
\hline \multicolumn{9}{|l|}{ Eje $\mathrm{Z}$} & \multirow[t]{8}{*}{0,68} & \multirow[t]{8}{*}{0,01} \\
\hline Semana 1 & 61 & $-1,45$ & $-0,70$ & $-0,30$ & $-0,28$ & 0,10 & 1,23 & 0,59 & & \\
\hline Semana 2 & 55 & $-2,10$ & $-0,60$ & $-0,20$ & $-0,23$ & 0,25 & 1,20 & 0,70 & & \\
\hline Semana 3 & 57 & $-2,60$ & $-0,80$ & $-0,10$ & $-0,32$ & 0,30 & 1,40 & 0,91 & & \\
\hline Semana 4 & 47 & $-2,00$ & $-0,70$ & $-0,20$ & $-0,29$ & 0,05 & 1,30 & 0,72 & & \\
\hline Semana 5 & 40 & $-2,00$ & $-0,62$ & $-0,15$ & $-0,23$ & 0,30 & 1,40 & 0,81 & & \\
\hline Semana 6 & 54 & $-1,90$ & $-0,60$ & $-0,10$ & $-0,18$ & 0,18 & 2,00 & 0,81 & & \\
\hline Semana 7 & 40 & $-2,80$ & $-0,52$ & $-0,15$ & $-0,13$ & 0,50 & 1,40 & 0,92 & & \\
\hline
\end{tabular}




\begin{tabular}{|c|c|c|c|c|c|c|c|c|c|}
\hline & $\mathrm{N}$ & Min & C0.25 & mediana & media & c0.75 & $\max$ & d.e. & $p$ \\
\hline \multicolumn{10}{|l|}{ Vejiga axial } \\
\hline Semana 1 & 59 & 5.30 & 7.85 & 8.93 & 8.99 & 9.87 & 12.52 & 1.39 & \multirow{8}{*}{0.16} \\
\hline Semana 2 & 54 & 3.33 & 7.23 & 8.25 & 8.47 & 9.75 & 13.02 & 1.85 & \\
\hline Semana 3 & 56 & 3.40 & 7.29 & 8.59 & 8.52 & 9.73 & 12.78 & 1.74 & \\
\hline Semana 4 & 46 & 5.02 & 7.54 & 8.74 & 8.66 & 9.73 & 11.99 & 1.65 & \\
\hline Semana 5 & 37 & 3.60 & 7.87 & 8.58 & 8.35 & 9.49 & 11.90 & 1.88 & \\
\hline Semana 6 & 52 & 3.74 & 6.68 & 8.00 & 8.11 & 9.00 & 12.40 & 1.89 & \\
\hline Semana 7 & 37 & 4.80 & 7.53 & 8.48 & 8.33 & 9.10 & 12.20 & 1.67 & \\
\hline Semana 8 & 9 & 4.53 & 7.12 & 8.10 & 8.04 & 8.27 & 10.70 & 1.86 & \\
\hline \multicolumn{9}{|c|}{ Vejiga coronal } & \multirow{9}{*}{0.48} \\
\hline Semana 1 & 59 & 5.07 & 6.85 & 7.47 & 7.53 & 8.14 & 9.94 & 0.97 & \\
\hline Semana 2 & 54 & 2.34 & 6.53 & 7.00 & 7.15 & 7.93 & 9.98 & 1.20 & \\
\hline Semana 3 & 54 & 2.80 & 6.59 & 7.30 & 7.22 & 7.95 & 10.53 & 1.25 & \\
\hline Semana 4 & 46 & 4.47 & 6.15 & 7.17 & 7.10 & 7.97 & 9.80 & 1.16 & \\
\hline Semana 5 & 37 & 4.80 & 6.40 & 7.00 & 7.11 & 7.60 & 9.60 & 1.09 & \\
\hline Semana 6 & 50 & 4.73 & 6.42 & 6.97 & 6.98 & 7.65 & 9.60 & 0.99 & \\
\hline Semana 7 & 37 & 4.55 & 6.60 & 7.33 & 7.20 & 7.93 & 8.73 & 0.97 & \\
\hline Semana 8 & 9 & 6.07 & 6.60 & 7.00 & 7.03 & 7.23 & 8.20 & 0.64 & \\
\hline \multicolumn{9}{|c|}{ Vejiga sagital } & \multirow{9}{*}{0.23} \\
\hline Semana 1 & 59 & 2.69 & 4.59 & 5.40 & 5.51 & 6.34 & 10.33 & 1.45 & \\
\hline Semana 2 & 54 & 1.06 & 4.15 & 5.08 & 5.28 & 6.18 & 9.66 & 1.72 & \\
\hline Semana 3 & 56 & 1.14 & 3.80 & 4.80 & 5.14 & 6.15 & 11.37 & 1.88 & \\
\hline Semana 4 & 46 & 2.13 & 4.20 & 5.63 & 5.38 & 6.38 & 9.90 & 1.68 & \\
\hline Semana 5 & 36 & 1.47 & 4.05 & 5.20 & 5.09 & 6.02 & 10.80 & 1.66 & \\
\hline Semana 6 & 52 & 2.13 & 3.65 & 4.44 & 4.84 & 6.11 & 9.50 & 1.70 & \\
\hline Semana 7 & 37 & 2.27 & 3.87 & 4.73 & 4.77 & 5.30 & 9.60 & 1.56 & \\
\hline Semana 8 & 9 & 1.61 & 3.30 & 3.87 & 4.37 & 6.00 & 7.90 & 1.93 & \\
\hline \multicolumn{9}{|l|}{ Recto axial } & \multirow{9}{*}{0.35} \\
\hline Semana 1 & 61 & 1.60 & 2.90 & 3.29 & 3.30 & 3.62 & 4.38 & 0.55 & \\
\hline Semana 2 & 55 & 2.00 & 2.60 & 3.07 & 3.10 & 3.49 & 6.00 & 0.72 & \\
\hline Semana 3 & 57 & 2.00 & 2.73 & 3.20 & 3.25 & 3.67 & 5.73 & 0.78 & \\
\hline Semana 4 & 47 & 1.73 & 2.70 & 3.20 & 3.21 & 3.65 & 4.60 & 0.68 & \\
\hline Semana 5 & 40 & 2.00 & 2.80 & 3.10 & 3.33 & 3.68 & 5.33 & 0.79 & \\
\hline Semana 6 & 54 & 1.80 & 2.66 & 3.34 & 3.33 & 3.97 & 6.40 & 0.89 & \\
\hline Semana 7 & 40 & 2.00 & 2.98 & 3.40 & 3.35 & 3.62 & 5.00 & 0.71 & \\
\hline Semana 8 & 9 & 2.00 & 2.53 & 2.93 & 3.21 & 3.40 & 5.20 & 1.04 & \\
\hline
\end{tabular}

